\title{
Editorial: Introduction to the Supplement on HIV, HAART, and Fertility in sub-Saharan Africa
}

\author{
Angela Kaida · David R. Bangsberg • \\ Glenda Gray · Robert S. Hogg • Rachel King • \\ Cari L. Miller
}

Published online: 12 May 2009

(C) Springer Science+Business Media, LLC 2009

Recent years have witnessed an unprecedented global effort aimed at providing universal access to highly active antiretroviral therapy (HAART), however, comparatively little attention has been given to how HAART may affect reproductive decision-making, sexual and reproductive practices, and fertility. ${ }^{1}$ These issues are particularly critical in HIV endemic settings where the majority of new infections occur among women (UNAIDS 2008).

\section{A. Kaida $(\bowtie) \cdot$ R. S. Hogg}

BC Center for Excellence in HIV/AIDS, 608-1081 Burrard Street, Vancouver, BC, Canada e-mail: akaida@interchange.ubc.ca

\author{
A. Kaida \\ School of Population \& Public Health, The University \\ of British Columbia (UBC), Vancouver, BC, Canada \\ D. R. Bangsberg \\ Harvard Medical School/MGH, Boston, MA, USA \\ G. Gray \\ Perinatal HIV Research Unit, University of the Witwatersrand, \\ Johannesburg, South Africa \\ R. S. Hogg · C. L. Miller \\ Simon Fraser University, Burnaby, BC, Canada \\ R. King \\ Global Health Sciences, University of California, \\ San Francisco (UCSF), San Francisco, CA, USA \\ R. King \\ Karolinska Institute, Stockholm, Sweden
}

The goal of this supplement of AIDS and Behavior is to bring together current, innovative research from sub-Saharan Africa that explores the influence of HAART on reproductive decision-making, reproductive and sexual health, and reproductive outcomes at individual, social, and environmental levels.

The concept for this supplement emerged from discussions at a public forum and research conference in Soweto and Johannesburg, South Africa on April 21-23, 2008 entitled: Switching gears in HIV research: Building an international agenda on the impact of highly active antiretroviral therapy (HAART) on fertility in sub-Saharan Africa (Forrest and Kaida 2008). The purpose of the conference was to foster international knowledge exchange on emerging research and policy priorities related to reproductive and sexual health (RSH), fertility decision-making, and fertility of HIV-positive individuals, during a time of rapidly expanding access to HAART. The 3-day event brought together internationally renowned HIV/AIDS and reproductive and sexual health stakeholders, including researchers, clinicians, policy-makers, community activists, and representatives of international development and health research funding agencies. As the conference drew to a close, it became clear that although exciting and important research was being conducted on the topic, there was a dearth of published literature available. As such, a commitment was advanced to support local researchers and conference delegates to develop their research findings into manuscripts and to circulate a broader international call for related papers on the topic of HIV, HAART, and fertility in

\footnotetext{
${ }^{1}$ Please note that the term fertility is being used in the demographic sense, as in the actual level of reproduction based on the number of live births that occur, rather than the biologic capability to reproduce (termed fecundity).
} 
sub-Saharan Africa. The end result of this commitment is this supplement. In line with the original intentions, the supplement provides research findings that can be used towards the design and implementation of evidence-based reproductive and sexual health policies and programs that support women, their families, and their communities in reproductive decision-making in the context of HIV.

The 13 papers herein include a mix between those presented at the conference and those solicited from a broader audience of international researchers. The papers reflect a wide array of issues related to HIV, HAART, and fertility from across sub-Saharan Africa. Taken collectively, the papers reveal fascinating epidemiological, behavioral, and institutional associations between HIV and fertility and explore the important ways HAART scale-up in HIV endemic regions is impacting reproductive decision-making, fertility, and aspects of HIV care and reproductive health services.

The papers address three fundamental challenges for HIV-affected couples who wish to have children: (1) Maintaining maternal heath before, during, and after the pregnancy; (2) Preventing mother-to-child transmission of HIV (PMTCT); and (3) Preventing HIV transmission to a sero-negative partner within discordant couples. This collection of papers, and by extension this supplement, discuss these challenges within a framework that recognizes and supports the sexual and reproductive health rights of people living with HIV.

The first paper by Matthews and Mukherjee provides a substantive review of the published literature regarding strategies to reduce the risks of HIV transmission to a seronegative partner among HIV-discordant couples in resource-limited settings where HAART is available. The authors introduce the concept and terminology of "harm reduction" as a supportive component within fertility counseling in HIV treatment and care services. The goal of a harm reduction approach is to first understand the fertility goals of the couple, and then design strategies that both meet that goal and minimize risks to the couple and their child.

The subsequent four papers provide important insight into the fertility intentions of HIV-positive individuals and whether access to HIV treatment services influences their intentions. This information is critical to inform the design and implementation of RSH services and policies that mitigate the risks associated with reproduction among HIV-affected couples.

To begin, the study by Yeatman longitudinally explored the question of whether discovering one's HIV status influences the desire to have children in rural Malawi. Beginning with a group of men and women who didn't know their HIV status, they found that, upon follow-up, respondents who received a positive test reduced their childbearing desires. Interestingly, only those who were surprised by their test result altered their fertility preferences, a finding which held true for both positive and negative results. This finding highlights the importance of individuals' perceptions about their own risk of HIV in mediating the impact of known HIV status on fertility intentions.

Taulo and colleagues use longitudinal data to compare the fertility intentions of HIV-positive and HIV-negative women in an urban setting in Malawi. They report that fertility intentions of HIV-positive women were lower than those of negative women and that over time, HIV-positive women were significantly more likely to change from wanting more children to not wanting more children. Importantly, however, despite lower and declining fertility intentions over time among HIV-positive women, the incidence of pregnancy did not differ between HIV-positive and HIV-negative women.

Both the Taulo et al. and Yeatman studies were conducted before population-level access to HAART was available in Malawi and, as such, reveal an association between HIV status and fertility intentions in the absence of HIV treatment services. In contrast, the Maier et al. study assessed the association between use of antiretroviral therapy and fertility desires among HIV-positive women in Uganda where population-level HAART is available. This study showed that HIV-positive women on HAART were three times more likely to desire (more) children, compared with HIV-positive women who were not on HAART. Interestingly, however, although use of HAART was associated with an increase in fertility desire, it was associated with a significantly decreased incidence of pregnancy and live birth.

Researchers from South Africa asked a similar question about the association between fertility intentions and use of antiretroviral therapy. Importantly, however, Cooper and colleagues included both men and women in their study and report salient gender-related differences. Namely, use of antiretroviral therapy was associated with higher fertility intentions among HIV-positive women (consistent with the findings from Uganda) but not among HIV-positive men.

Collectively, the findings of these four studies suggest that HIV-positive treatment naïve women have the lowest fertility intentions compared with HIV-negative women and HIV-positive women on HAART. An important question remains regarding whether the fertility intentions of HIVpositive women on HAART will begin to resemble the expressed fertility intentions of HIV-negative women. It is clear that much more needs to be understood about the factors influencing fertility intentions of HIV-positive men. In addition, in those two studies that investigated actual fertility outcomes (Taulo et al.; Maier et al.), despite significant differences in fertility intentions, neither found 
differences in incidence of pregnancy or livebirths. This finding may reflect the recency of HAART availability in the region and on-going research in this area will be critical.

The next set of studies, both from South Africa, use qualitative data to explore more in-depth aspects of fertility decision-making. The first, by Laher and colleagues, presents an analysis of the effect of HIV and HAART on pregnancy desires and contraceptive use among HIV-positive women. The authors report that stated fertility desires of HIV-positive women are highly shaped by fears and considerations around the disease and disease progression. Contraceptive choices, however, are influenced by many of the same concerns expressed by HIV-negative women, including real and normative side effects and the mitigating influence of partnership status. Forrest et al.'s study qualitatively investigates the perceptions of adolescents towards childbearing among HIV concordant and discordant couples. Adolescents expressed a high level of personal and societal stigma towards HIV-affected couples who wish to conceive. The adolescents also spoke to a lack of youthfriendly HIV and RSH services in Soweto and noted a number of barriers associated with accessing services. The importance of addressing adolescent reproductive and sexual health needs cannot be overstated since in many regions, HIV incidence is increasing most dramatically among young people aged 18-24 years (UNAIDS 2008), which coincides with the initiation and peak reproductive years (Population Division of the Department of Economic and Social Affairs of the United Nations Secretariat 2008).

The next two papers move away from investigating fertility intentions, to exploring HIV-positive women's concerns about the safety of childbearing. Nduna and Farlane report results from a qualitative study regarding the questions HIV-positive women in South Africa have pertaining to living with HIV, being on HAART, and becoming pregnant. The women expressed an urgent need for nonstigmatizing and non-judgmental HIV and RSH services that support their rights to safely achieve their fertility goals. One of the recurrent questions raised by women in Nduna and Farlane's study pertained to the influence of pregnancy on HIV disease progression in the context of HAART use. In the absence of clear and synthesized available evidence, MacCarthy and colleagues set out to respond to this question in a literature review. They report that there appears to be no negative effect of pregnancy on HIV disease progression. Moreover, recent studies from high-income countries (albeit few in number) suggest that pregnancy may positively modify the HAART response.

The final set of supplement papers discuss a variety of social, structural, and institutional factors related to HIV, HAART, and fertility that are of pressing importance as HAART scale-up expands in HIV endemic regions.
The paper by Kaida et al. explores the issue of HAART optimism as it relates to women and fertility. They present a validated and reliable scale, termed the Women's HAART Optimism Monitoring and Evaluation scale (WHOMEN's scale), which may be valuable to future studies interested in assessing the prevalence of HAART optimism and it's impact on reproductive intentions and sexual behaviors of HIV-positive women in HIV endemic settings.

Byakika-Tusiime and colleagues found that providing family-based treatment in Uganda (i.e., treatment to all HIVinfected members of a household) is associated with excellent adherence in both the HIV-infected parents and their HIV-infected children, but that depression in the post-natal period may be a vulnerable time for sustained adherence. Theuring et al. explores male involvement in the reproductive health services and antenatal care (ANC) process of their HIV-positive partners in Tanzania. The authors found that men were positively inclined to participate in ANC/PMTCT services but that external social and institutional barriers play an important role in preventing them from doing so. The final paper by Agadjanian and Hayford, uses an institutional perspective to analyze how Maternal and Child Health units, where voluntary counseling and testing, PMTCT, and HAART delivery services have been integrated with traditional services, play a critical role in the connection between HAART rollout and reproductive behavior.

In sum, the 13 studies included in this supplement present a broad picture of the current state of research on issues related to HIV, HAART and fertility in sub-Saharan Africa. The studies call for additional related research to inform HIV care and RSH services that address the diverse reproductive needs of HIV-positive populations and HIVnegative individuals living in endemic settings. In particular, additional work is necessary to support the rights of HIV-positive individuals to be sexually active and achieve their fertility goals, while minimizing the risk of HIV transmission. It is our hope that the supplement has contributed one step toward achieving these critical global health priorities.

In conclusion, we would like to acknowledge the contributions of others in making the publication of this supplement possible. Funding for the conference (and some of the presented research findings) was provided by Canada's Social Science and Humanities Research Council (SSHRC), the Office of the Vice President Academic at Simon Fraser University, the Canadian Foundation for AIDS Research (CANFAR), the Council for AIDS Research (CFAR), the Perinatal HIV Research Unit (PHRU), and the British Columbia Center for Excellence in HIV/AIDS (BCCfE). Funding for this supplement was provided by the International Program of the Harvard University Center for AIDS Research. 


\section{References}

Forrest, J., \& Kaida, A. (2008). Conference summary report: Switching gears in HIV research: Building an international research agenda on the impact of HAART on fertility in subSaharan Africa. Soweto and Johannesburg, South Africa, Perinatal HIV Research Unit (PHRU). Available at: http://www. phru.co.za/pdf/conference/joburg_conference_summary_report_ may30_2008.pdf. Accessed 1 April 2009.
Population Division of the Department of Economic and Social Affairs of the United Nations Secretariat. (2008). World population prospects: The 2008 revision. United Nations Secretariat.

UNAIDS. (2008). Report on the global HIV/AIDS epidemic 2008. Geneva: UNAIDS. 